\title{
Linearly Polarized Mode Division Multiplexed Transmission over Ring-Index Multimode Fibres
}

\author{
X.Q. Jin, R. Li, D.C. O'Brien, and F.P. Payne \\ University of Oxford, Parks Road, Oxford, OX1 3PJ, UK \\ Email: xianqing.jin@eng.ox.ac.uk
}

\begin{abstract}
LP modes in ring-index multimode fibres (RIMMFs) are proposed to carry parallel-data for spatial multiplexing systems with low-complexity MIMO processing. Simulation shows that, with $3 \times 3$ MIMO, $6 \times 20 \mathrm{~Gb} / \mathrm{s}$ NRZ errorfree transmission is achieved over $1 \mathrm{~km}$-RI-MMFs using IMDD.
\end{abstract}

\section{INTRODUCTION}

To satisfy the requirements of increased capacity of future systems, a parallel data transmission using multiple optical paths, called spatial multiplexing or space-division multiplexing (SDM) was recently proposed [1]. In SDM systems, multiple-input and multiple-output (MIMO) processing is required in the receivers to compensate linear cross talk between optical modes due to mode coupling [2-3]. The complexity of MIMO depends on the length of impulse response and the number of transmitters/receivers requiring MIMO processing. In practice, the length of impulse response can be easily reduced using differential group delay compensation. Therefore, the number of transmitters/receivers requiring MIMO processing plays a key role in determining the MIMO complexity. To reduce the complexity of SDM systems, mode coupling can be minimized to avoid the use of MIMO processing in the receivers [4]. In such SDM systems without MIMO processing, however, capacity/spectral efficiency is limited by the small number of modes carrying data signals in the optical fibres [4].

Ring-index multimode fibres (RI-MMFs) were proposed for fibre-optic communication in 1970s [5]. Orbital angular momentum (OAM) modes in RI-MMFs have been reported to be able to carry independent data streams in SDM systems [6]. In this paper, linearly polarized (LP) modes in RI-MMFs are proposed for SDM transmission with low complexity MIMO processing because of their key feature: the propagation constant difference between two adjacent LP modes, $\Delta \beta$, which is inversely proportional to mode coupling coefficient [7-8], significantly increases with increasing azimuthal number of LP modes for the same radial mode number. In the RI-MMF-based SDM system, MIMO can be only used to recover signals on the low order LP modes with strong mode coupling rather than high order LP modes with negligible mode coupling for a certain transmission distance. Using $3 \times 3$ MIMO with six transmitters/receivers, both theoretical and numerical investigations are undertaken of $6 \times 20 \mathrm{~Gb} / \mathrm{s}$ NRZ signals transmission over $1 \mathrm{~km}$ RI-MMFs using intensity modulation/direct detection (IMDD).

\section{RING-INDEX MULTIMODE FIBRES AND MODE COUPLING}

As illustrated in Fig. 1(a), the refractive index profile of a RI-MMF has a step ring layer with thickness of $d$ at an average radius of $r_{a}$. The refractive index in the ring (cladding) layer is $n_{1}\left(n_{2}\right)$. It is noted that the ring-index profile can be optimized for the desired mode propagation constant, $\beta$. As a simple example, a step ring-index profile is adopted here. By numerically solving the scalar wave equation with RSoft (ModeSYS), electrical fields, propagation constant and relative mode delay of $L P_{l m}$ modes can be obtained. $l$ and $m$ represent the azimuthal and radial number of each optical mode, respectively. Fig. 1(b) shows intensity profiles of modes LP01, LP11a, LP02, LP22a, which are well located in the ring layer without spreading power into cladding layers.
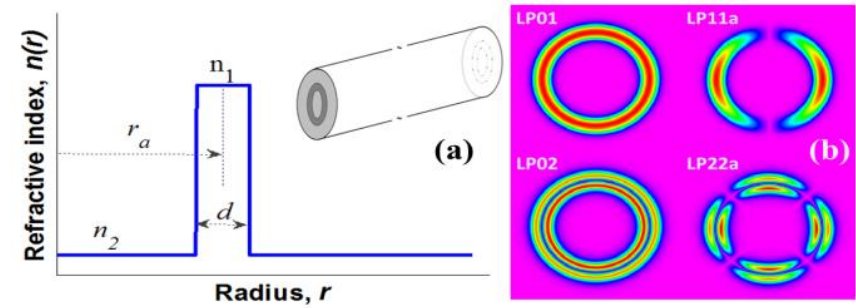

Fig. 1. (a) Refractive index profile and (b) LP modes intensity profile
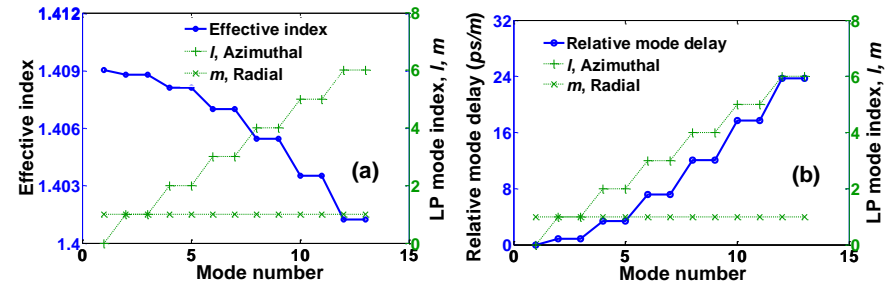

Fig. 2. (a) Effective index and (b) Relative mode delay, $r_{a}=10 \mu \mathrm{m}, d=3.8 \mu \mathrm{m}$, $\lambda=1550 \mathrm{~nm}$. A pair of LP modes with the same $l, m$ number represents two degenerate modes $\mathrm{LP}_{l m a, b}$.

Theoretically, the maximum azimuthal number of guided modes increases with increasing radius and thickness of the ring layer, while the maximum radial number of guided modes only increases with thickness of ring layer [9]. It means that the number of modes can be varied by adjusting the radius and thickness of ring layer. Because guided modes' effective index (EI) equal to $\beta / k_{0}\left(k_{0}\right.$ : wave number) is distributed in the range from $n_{1}$ to $n_{2}$, there is a trade-off between large number of guided modes and large $\Delta \beta$. To efficiently use the guided modes with large $\Delta \beta$ for SDM transmission, all guided modes with radial mode number of one in the RI-MMFs are preferred. From both simulation and theoretical analysis, the cut-off frequency for $L P_{l m}(m=1)$ modes is $\leq 3.14$, which corresponds to a RI-MMF with a thickness of about $3.8 \mu m$ at $r_{a}=10 \mu \mathrm{m}$ and NA of $0.2\left(n_{2}=1.4\right)$ for a wavelength of $\lambda$ $=1550 \mathrm{~nm}$. Therefore, RI-MMFs with $r_{a}=10 \mu \mathrm{m}$ and $d=3.8 \mu \mathrm{m}$ are used in the following simulations. Fig. 2(a,b) plots effective index and relative mode delay (group delay) as a 
function of mode number corresponding to $L P_{l m}$ modes in the RI-MMFs. The value of EI (relative mode delay) decreases (increases) with increasing mode number.
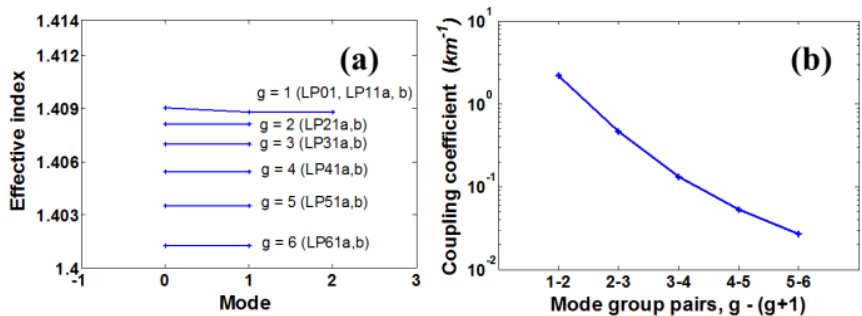

Fig. 3. Effective index of modes in the mode group $g$ and (b) Coupling coefficient between two neighbouring mode groups

To study the mode coupling effect in RI-MMFs, the coupled power equation derived by Marcuse is used [10]. The power of mode $n, P_{n}$, can be described below as function of time $t$ and position $z$ along the fibre axis.

$$
\frac{d P_{n}}{d z}+\tau_{n} \frac{d P_{n}}{d t}=-\gamma_{n} P_{n}+\sum_{m} d_{n m}\left(P_{m}-P_{n}\right)
$$

where $\tau_{n}$ is group delay per unit length of mode $n, \gamma_{n}$ is the mode power attenuation coefficient. $d_{n m}$ is the mode coupling coefficient between mode $n$ and mode $m$. The mode coupling coefficient can be well approximated in the high frequency region of the curvature spectrum by

$$
d_{n m} \approx \frac{A}{\left(\beta_{m}-\beta_{n}\right)^{2 p}}
$$

where $A$ is the coupling strength parameter. The value of $p$, which depends on the model studied, is typically equal to 2 [8]. The $A$ is set to $2 \times 10^{11}$, which corresponds to a measured average coupling coefficient of $0.15 \mathrm{~km}^{-1}$ for $62.5 \mu \mathrm{m}$ MMFs (108 Cambridge MMFs) [11]. Eq. (1) can be numerically solved by a split-step method, in which mode coupling and mode propagation steps are alternated in each axial step $\Delta z$. The step $\Delta z$ must be smaller than inverse value of maximum $d_{n m}$ for accurate results.

In RI-MMFs, there exist some modes with approximately the same value of $\beta$. These modes are assumed to experience complete mode coupling for very small $\Delta \beta$. By defining a constant of $\varepsilon=10^{3}$ [8], the modes in Fig. 2(a) with $\Delta \beta<\varepsilon$ can be grouped into mode groups as shown in Fig. 3 (a). As the number of modes in the RI-MMFs is small, the coupled equation for mode groups derived by assuming a large number of modes in MMFs in $[7,8,11]$ is not valid here. The coupling coefficient between two mode groups is calculated using the average $\beta$ of modes in a mode group by Eq. (2). Fig. 3(b) shows coupling coefficient, $d_{g}$, between mode group $g$ and $g+1$. The value of $d_{g}$ significantly decreases with mode group number, indicating that signals on the low order modes with large mode coupling require MIMO to compensate the linear cross talk, while signals on the high order modes with small mode coupling may not need MIMO.

\section{TRANSMISSION PERFORMANCE}

Based on the calculated relative mode delay in Fig. 2(b) and mode coupling coefficient in Fig.3(b), investigation is made of $6 \times 20 \mathrm{~Gb} / \mathrm{s} \mathrm{NRZ}$ signals in a $1 \mathrm{~km}$ RI-MMF-based SDM system with 6 transmitters/receivers using IMDD. Each $20 \mathrm{~Gb} / \mathrm{s}$ NRZ signal propagates on a mode group. $3 \times 3 \mathrm{MIMO}$ is used in the transmitters/receivers 1-3 to mitigate the effect of strong mode coupling between mode groups 1-3. It is assumed here that 1) the differential mode attenuation in Eq. (1) is ignored for short distance transmission, 2) ideal IMDD, mode converters (for example, spatial light modulators) and mode Mux/Demux are used, and 3) only additive white Gaussian noise (AWGN) in the receiver is considered. The optical power coupled into each mode group is equal to $0 \mathrm{dBm}$.

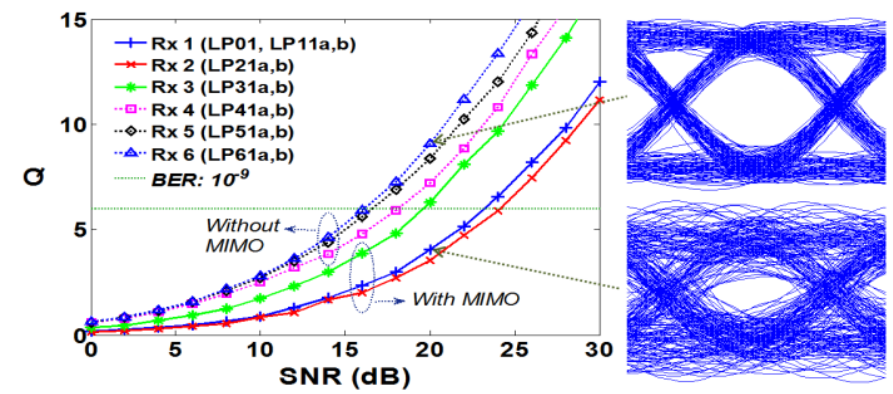

Fig. 4. Q Versus SNR (1km) and Eye diagrams in receivers 1 and 6

Fig. 4 shows the transmission performance (Q for BER estimation) of $6 \times 20 \mathrm{~Gb} / \mathrm{s}$ NRZ signals over $1 \mathrm{~km}$ RI-MMFs as a function of SNR and eye diagrams in receivers 1 and 6 . Error-free transmission of all NRZ signals can be achieved for SNRs $>24 \mathrm{~dB}$. As a small amount of power is coupled between relatively high order modes and relatively low order modes, the required average SNR for BER of $10^{-9}$ for receivers 4-6 (without MIMO) is about $4.5 \mathrm{~dB}$ less than that for receivers 1-3 (with MIMO). For the receivers 4-6, the value of Q increases with increasing the mode order because of less mode coupling on the relatively high order modes. As half of receivers do not use MIMO, the complexity of SDM with MIMO is significantly reduced by $75 \%$ (calculated by $\left(K^{2}-(K / 2)^{2}\right) / K^{2}$, where $K$ is number of transmitters/receivers).

\section{CONCLUSIONS}

It's shown that RI-MMFs have great potential for reducing the complexity of SDM systems with MIMO which is used in the half of all receivers. The radius and thickness of ring layer in RI-MMFs can be optimized depending on requirements of system capacity and transmission distance.

\section{ACKNOWLEDGEMENT}

We gratefully acknowledge RSoft for providing the software support. This work was supported by EPSRC-funded project (grant number: EP/J008745/1).

\section{REFERENCES}

[1] H.R. Stuart, Science, vol. 289, pp. 281-283 (2000).

[2] R. Ryf, et al, J. Lightwave Technol., vol. 30 (4), pp. 521-531 (2012)

[3] B.C. Thomsen, et al, OFC/NFNEC 2010, p. OThM6

[4] N. Hanzawa, et al, OFC/NFNEC 2011, p. OWA4

[5] R.H. Stolen, Applied Optics, vol. 14 (7), pp.1533-1537 (1975)

[6] N. Bozinovic, et al, ECOC 2012, p. TH.3.C.6

[7] R. Olshansky, Applied Optics, vol. 14 (4), pp.935-945 (1975)

[8] D. Yevick, et al, Applied Optics, vol. 22 (7), pp.1010-1015 (1983)

[9] J. Marcou, et al, Microw.\&Opt. Tech. Lett. vol.38(3), pp.249-254 (2003)

[10] D. Marcuse, Bell Syst. Tech. J., vol. 51, pp.229-237, (1972)

[11] K. Balemarthy, et al, J. Lightwave Technol., vol. 24 (12), pp.4885-4894 (2006). 